\title{
In vivo Differentiation Potential of Mesenchymal Stem Cells: Prenatal and Postnatal Model Systems
}

\author{
Courtney Quinn Alan W. Flake \\ The Children's Center for Fetal Research, The Children's Hospital of Philadelphia, Philadelphia, PA, USA
}

\section{Key Words}

Mesenchymal stem cells - Differentiation capacity .

Prenatal model systems · Postnatal model systems

\section{Summary}

Most of our knowledge of mesenchymal stem cell (MSC) biology is derived from in vitro systems that are often highly contrived to favor culture expansion or specific differentiation events. However, any conclusions drawn from in vitro studies regarding MSC differentiation capacity, immune properties, or therapeutic potential must be validated by in vivo studies to ultimately be meaningful. At the present time, there are relatively few in vivo studies demonstrating differentiation and functional integration of MSCs into host tissues after transplantation. There is a need for in vivo model systems to assay MSC biology and to move potential therapeutic strategies forward. Here, we review prenatal model systems as potentially advantageous for the in vivo characterization of MSCs, and we critically review the results of in vivo studies of MSC transplantation in prenatal and postnatal model systems with an emphasis on proven engraftment and differentiation.

\section{Introduction}

The isolation of mesenchymal stem cells (MSCs) from many mesoderm derived tissues, their relative ease of culture expansion, and their multipotentiality in vitro, suggests the potential

\section{Schlüsselwörter}

Mesenchymael Stammzellen · Differenzierungskapazität · Pränatale Modelsysteme · Postnatale Modellysteme

\section{Zusammenfassung}

Unser Wissen über die Biologie mesenchymaler Stammzellen (MSCs) basiert hauptsächlich auf In-vitro-Systemen, welche oft auf sehr künstliche Art und Weise das Kulturwachstum oder spezifische Differenzierungsvorgänge begünstigen. Aus In-vitro-Studien gezogene Schlussfolgerungen bezüglich der Differenzierungskapazität, der Immuneigenschaften oder des therapeutischen Potentials von MSCs müssen jedoch durch In-vivoStudien bestätigt werden, um aussagekräftig sein zu können. Im Moment gibt es relativ wenige In-vivo-Studien, die die Differenzierung und funktionelle Integration von MSCs in das Gewebe des Empfängers nach einer Transplantation demonstrieren. Es besteht Bedarf an In-vivoModellsystemen, mit denen die MSC-Biologie geprüft und potentielle therapeutische Strategien gefördert werden können. Wir geben einen Überblick über pränatale Modellsysteme, die für die In-vivo-Charakterisierung von MSCs potentiell von Vorteil sein könnten, und bewerten kritisch die Ergebnisse von In-vivo-Studien zur Transplantation von MSCs in prä- und postnatalen Modellsystemen mit Betonung auf nachgewiesenem Anwachsen und Differenzierung der Zellen.

\begin{tabular}{ll}
\hline KARGER & @ 2008 S. Karger GmbH, Freiburg \\
$\begin{array}{l}\text { Fax +49761 4520714 } \\
\begin{array}{l}\text { E-mail Information@Karger.de } \\
\text { www.karger.com }\end{array}\end{array}$ & $\begin{array}{l}\text { Accessible online at: } \\
\text { www.karger.com/tmh }\end{array}$ \\
&
\end{tabular}

Alan W. Flake, M.D

The Children's Institute for Surgical Science

Department of Surgery, Abramson Research Center, Rm 1116B

3615 Civic Center Blvd, Philadelphia, PA 19104-4318, USA

Tel. +1 215 590-3671, Fax -3324

E-mail flake@email.chop.edu 
ic MSCs are not successfully engrafted using current protocols for hematopoietic stem cell transplantation (HSCT), with MSCs and their stromal derivatives remaining primarily of host origin in the majority of rigorous studies [4, 5]. Many in vivo studies of site-specific or systemic administration of culture expanded MSCs have now been published. However, relatively few have clearly demonstrated MSC engraftment with differentiation and functional incorporation into the recipient tissues when subjected to critical review. In many instances, the beneficial effects can be attributed to paracrine bystander effects rather than a direct participation of MSCs in tissue repair. This discrepancy between in vitro and in vivo evidence of MSC differentiation and multipotentiality highlights the need for model systems for MSC transplantation that are permissive for both engraftment and differentiation.

\section{Prenatal Model Systems for Mesenchymal Stem Cell Transplantation}

Prenatal model systems theoretically offer several unique advantages for in vivo assessment of the engraftment and differentiation potential of MSC populations. The primary advantage is the immature immune status of the fetus that is permissive for engraftment of allogeneic and xenogeneic cells, removing the confounding factor of immune response. In addition, the early gestational environment is the only time in life when large scale migrations of stem cells occurs to form tissue compartments. Thus, the opportunity may exist to engraft MSCs in a more physiologic way, without the need for ablative measures or tissue injury. The proliferative milieu of the early gestational fetus may facilitate expansion of donor cells after engraftment, and regulatory processes in the fetal environment may provide specific inductive signals for differentiation. Finally, the ability to engraft xenogeneic cells may provide a powerful tool to analyze species-specific gene expression permitting in situ functional analysis. Finally, the prenatal therapeutic potential of MSCs for correction of specific disorders provides an impetus for pre-clinical studies in fetal models.

A first hint that the prenatal environment might be permissive for MSC engraftment came from the observation of AlmeidaPorada et al. [6] who observed that the bone marrow (BM) of sheep previously transplanted in utero with human hematopoietic stem cells (hHSCs) contained a population of cells that were capable of forming human stromal elements in vitro. This observation led to subsequent studies in which cultured stromal elements were co-transplanted with hematopoietic cells $[7,8]$ with a goal of facilitating hematopoietic engraftment. The co-transplantation of allogeneic adult or fetal BMderived stromal elements, with adult or fetal hematopoietic cells, into pre-immune fetal sheep markedly increased donor cell chimerism in all groups at 60 days post-transplantation, as assessed by peripheral blood (PB) analysis of chimerism. Ad- ditionally, stromal cell co-transplantation resulted in increased donor cell engraftment in the BM, with the long-term effect of increased donor hematopoiesis. A subsequent similar study utilizing the pre-immune human into sheep xenotransplantation model demonstrated that co-transplantation of hHSCs and BM-derived stromal cell progenitors led to enhanced long-term engraftment in the BM of chimeric sheep, as well as higher circulating levels of donor cells at an earlier timepoint than previously demonstrated [8]. The stromal cells utilized in these studies were not characterized as 'MSCs', and the purpose of the studies was not for assessment of MSC engraftment or differentiation potential. Nevertheless, these experimental findings were the first demonstration of functional engraftment of stromal cells for the support of hematopoiesis in vivo.

The first study to demonstrate the prenatal engraftment and differentiation of a well-defined population of MSCs was performed by Liechty et al. [9]. Human MSCs (hMSC) were successfully transplanted into fetal sheep and demonstrated longterm engraftment (up to 13 months). Additionally, the cotransplanted MSCs underwent site-specific differentiation into various mesenchymal cell lineages. In this study, no differentiation of hMSCs into non-mesoderm-derived tissues was identified, and the functional status of the engrafted cells could not be ascertained due to their low frequency. An interesting observation of this study was the persistence of human cells even when the transplants were performed relatively late in gestation, after the expected onset of immune competence. These early studies of prenatal transplantation of stromal elements and, subsequently, well characterized MSCs, confirmed the potential of prenatal models for in vivo studies of the engraftment, differentiation, and functional integration of MSCs.

The unique permissiveness of the fetal environment for MSCs has been further demonstrated in the human sheep model by a series of studies from investigators at the University of Nevada, Reno, that documented expanded in vivo differentiation capacity for MSCs derived from various sources. Specifically, Almeida-Porada et al. [10] transplanted human metanephric mesenchymal cells (MNMCs) into fetal sheep which resulted in engraftment and differentiation into functional hepatocyte-like cells, as well as hematopoietic cells. The latter population produced sustained engraftment and differentiation upon subsequent transplantation into secondary fetal sheep recipients. A few limitations of this study are noteworthy. First, although the human hepatocyte-like cells were functional (i.e. they produced albumin), no circulating levels of human albumin were found in the serum of the recipient sheep. In addition, the transplanted MNMCs were not clonal in derivation, but rather consisted of a heterogeneous population of cells, raising the possibility of derivation of the end-differentiated cells from multiple stem cell types. Therefore, the plasticity of a single cell was not demonstrated. 
More recently, Chamberlain et al. [11] examined whether different routes of administration of MSCs into fetal sheep affected their ability to engraft and differentiate into hepatocytes. Human BM MSCs expanded from a single cell were transplanted into fetal sheep via intrahepatic (IH) or intraperitoneal (IP) injection. Though both routes of administration led to MSC engraftment, the differentiation into functional human hepatocytes was significantly higher in the IH transplant group. Notably, the distribution of hepatocytes was found to be dependent on the route of administration; IP-injected animals exhibited a periportal distribution, whereas $\mathrm{IH}-$ injected animals demonstrated a more widespread distribution throughout the liver parenchyma. This group has previously demonstrated that MSCs transplanted into fetal sheep can differentiate into hematopoietic cells giving rise to hematopoietic engraftment $[12,13]$, raising the question of whether the MSCs or hematopoietic derivatives differentiated into hepatocytes. As the IH-injected animals had less hematopoietic engraftment but higher levels of donor hepatocytes, the study supported the direct differentiation of hMSCs rather than via a hematopoietic intermediary. The differentiation of functional hepatocytes was corroborated by the presence of human albumin in the serum, although the levels of human albumin were not as high as might be anticipated by the levels of engraftment observed. Unfortunately, the authors did not exclude fusion in this study as a mechanism for MSCs to hepatocyte differentiation.

The use of fetal tissue-derived MSCs might provide additional advantages for demonstration of engraftment and plasticity in fetal models. Fetal tissue-derived MSCs have been shown to have a higher proliferative capacity, express primitive stem cell markers, and have longer telomeres than adult MSCs [14]. Fetal tissue-derived MSCs from a variety of sources have now been utilized in studies in various fetal models. In the fetal sheep model, Airey et al. [15] have injected both adult and fetal hMSCs (intraperitoneally) into sheep fetuses. They demonstrated successful engraftment of cells into the hearts of fetal lambs, regardless of the source of MSCs (adult BM, fetal liver, or fetal brain). An interesting finding was that the vast majority of engrafted cells differentiated into Purkinje fibers of the ventricular conduction system; virtually no cells $(0.01 \%)$ differentiated into cardiomyocytes. A possible explanation for this finding provided by the authors was that the Purkinje fiber system may have been in an expansion phase during injection of the MSCs, thereby promoting their differentiation into Purkinje fibers. Alternatively, the transplanted MSCs may have preferentially differentiated into this specific cell type. In a subsequent study by the same group [16], hMSCs were shown to engraft and differentiate into cells with a cardiac phenotype. Here, fetal liver-derived MSCs isolated by Stro-1 selection (a marker of clonogenic BM stromal progenitor cells) were fluorescently labeled with one of two fluorescent dyes, and intraperitoneally injected into fetal sheep. hMSCs were found to engraft within 29-39 h post-transplanta- tion. Of particular interest was the finding that by $39 \mathrm{~h}$, engrafted cells no longer expressed Stro-1, but rather expressed a known early transcription factor of cardiogenesis, $\mathrm{Nkx} 2.5$. The majority of donor cells were found in the Purkinje fiber system of the heart with a low frequency of donor-derived cardiomyocytes documented. Interestingly, aggregates of Purkinje fibers contained either one dye or the other, indicating that each aggregate originated from the proliferation of a single MSC.

Other studies utilizing fetal MSCs have been performed in the murine model of in utero transplantation. Chan et al. [17] transplanted early gestational circulating hMSCs into dystrophic $(\mathrm{mdx})$ mice at E14-E16 gestation. They observed widespread, long-term engraftment with evidence of myogenic differentiation of MSCs into skeletal and myocardial muscle as well as other cell types including hepatocytes. The frequency of donor cells was very low (1-3/1,000 cells in non-muscle tissue, and 5-10/1,000 cells in muscle tissue), but the cells were convincingly demonstrated to be myocytes with expression of human dystrophin from some of the human nuclei. In a more recent study, the same group utilized the same source of MSCs to demonstrate improvement of phenotype in Type III Osteogenesis Imperfecta (OI) mice [18]. Transplanted cells were preferentially localized to areas of bone formation and repair, implicating osteogenic differentiation. Donor cells found in bone expressed osteoblast lineage genes and produced the extracellular bone structural protein osteopontin. Engraftment levels were around 5\% initially, similar to levels found in human studies of OI, but decreased over time. Nonetheless, over a $60 \%$ reduction in fracture incidence occurred, which led to a markedly improved disease phenotype. The duration of follow-up in this study was only 12 weeks raising the question of whether a true osteoblast repopulating cell was engrafted.

Efforts to demonstrate functional engraftment of adult MSCs after systemic administration in the fetal murine model have been less convincing. Chou et al. [19] used adult BM-derived hMSCs and transplanted them via IP injection into pre-immune, but immunocompetent fetal mice at E14. Results demonstrated low-level, intermediate-term engraftment (4 months). These investigators also found that donor-derived $\mathrm{CD} 45+$ cells were detectable in recipient $\mathrm{PB}$, suggesting the differentiation of MSCs into hematopoietic cells although the analysis of this observation was incomplete. Finally, an interesting study of site-specific MSC injection into the central nervous system (CNS) of fetal mice was published by MunozElias et al. [20]. They injected well characterized adult murine MSCs into the lateral ventricles of E15 mice and documented migration and neural differentiation of the cells after birth. In this non-injury, developmental model, the cells appeared to follow normal routes of neural migration and to differentiate in significant numbers into neural cells with anatomic integration into the CNS. 


\section{Postnatal Model Systems for Mesenchymal Stem Cell Transplantation}

\section{Systemic Administration}

While many claims have been made, the postnatal systemic administration of MSCs has been generally disappointing. The typical pattern is one of initial lodgment of most of the cells in the lungs with gradual, very low frequency distribution to most peripheral tissues as detected by PCR-based techniques. The exact nature of the persistent cells is unclear as in general, their frequency is too low for detailed characterization. A few studies of interest will be presented here, primarily to critically assess some of the claims that have been made.

Clinical studies utilizing MSC infusion have thus far yielded little information relevant to in vivo engraftment, multipotentiality, or function. A clinical study which is often cited to demonstrate the therapeutic effects of systemic administration of MSCs came from Horwitz et al. [21]. In this trial, pediatric patients with OI underwent allogeneic HSCT in an effort to attenuate the crippling abnormalities of this disorder. Transplanted BM cells were found to engraft and generate functional osteoblasts, which led to an improvement in bone structure and function. Though only short-term, low-level engraftment (1.5-2\%) was achieved, a follow-up study demonstrated continued improvements in biochemical, structural, and functional parameters over an extended period of 18-36 months post-transplant [22]. It is important to note that whole BM was transplanted, so inferences regarding the differentiation of MSCs into functional osteoblasts must be made with caution. Infusion of these patients with same donor MSCs 18-34 months after the HSCT protocol resulted in further benefit which was once again limited in duration [23]. These reports demonstrated short-term engraftment of donor osteoblasts, but long-term persistence was not determined. Interestingly, an experimental study in a postnatal lethal irradiation model from the same authors demonstrated osteoblast repopulation from the non-adherent fraction of BM with demonstration of a common progenitor for osteoblasts and hematopoietic cells in that fraction [21]. This raises some question regarding the stem cell responsible for maintenance of the osteoblast compartment and whether MSCs derived from the adherent fraction of BM are capable of reconstitution or long-term maintenance of the osteoblast compartment.

There have been a number of animal studies in which various MSCs have been infused systemically either with the intent of improving hematopoietic engraftment or for tracking and determination of cell fate. Bensidhoum et al. [24] studied the effects of subsets of hMSCs on the engraftment of hHSCs in a NOD/SCID mouse model. Either Stro-1-positive or Stro-1negative MSCs were co-transplanted with CD34+ cells. Stro-1negative (but not Stro-1-positive) MSCs resulted in significant increases in the percentage of hematopoietic cells in the BM of recipient mice. An interesting finding in terms of homing was demonstrated; Stro-1-positive cells migrated with better efficiency than Stro-1-negative cells to the BM, spleen, muscle, liver, and kidney, suggesting that pre-selection of MSCs for in vivo gene delivery may lead to improved tissue-specific homing of donor MSCs. While engraftment of donor MSCs was documented, differentiation was not assessed. The disconnection between homing and facilitation of hematopoietic engraftment for Stro-1 cells in this study suggests that the improvement in engraftment was related to cytokine influences on the hematopoietic graft rather than direct effects of donorderived stroma. Mahmud et al. [25] examined the effects of the route of administration of MSCs on engraftment in radiation-conditioned and non-conditioned adult baboons. Animals receiving either total body irradiation (TBI) or hemibody irradiation (HBI) were transplanted with MSCs by either intravenous (IV) or direct injection into BM cavities (intra-bone marrow, IBM). Animals that received donor MSCs via IV injection had significantly greater numbers of fibroblast colonyforming units (CFU-F) in the BM in both treatment groups than animals that did not receive any MSC transplant. Additionally, no significant improvements in engraftment in the IBM-transplanted group were demonstrated. The authors attributed this finding to the wide variation of CFU-F numbers seen in the animals transplanted by this route. In terms of cell fate, $\beta$-galactosidase-positive cells were detected in the BM of transplanted animals in both groups. No specific conclusions can be drawn from this study regarding the functional status of transplanted donor MSCs due to the low frequency of engrafted cells and lack of resolution of the methodology used in the study.

There have been a number of studies published utilizing murine 'MSC' populations that have been widely quoted as supportive of MSC engraftment and multipotentiality, but which contain significant methodologic flaws. It is now well recognized that murine MSCs require different isolation techniques than MSCs from other species due to the plastic adherence of murine hematopoietic cells. There have now been a number of protocols published which yield murine MSCs free of hematopoietic contamination. It is important in interpreting the literature to assess whether the murine population utilized was appropriately isolated and well characterized in this respect. For instance, Deng et al. [26] assessed a specific subpopulation of 'MSCs' $\left(\mathrm{flk}^{+}, \mathrm{sca}-\mathrm{1}^{-}\right)$in a co-transplantation experiment in which cultured allogeneic BM-MSCs and syngeneic BM cells were co-transplanted into lethally irradiated mice. Analysis at 150 days after transplant demonstrated mixed multilineage hematopoietic chimerism. They concluded that allogeneic MSCs could reconstitute hematopoiesis with induction of associated donor-specific tolerance. A more likely explanation given the isolation technique used and the minimal characterization of the MSC population utilized, was the presence of contaminating donor HSCs. Another example of hematopoietic contamination of murine MSC cultures confounding experimental results is the study of Niyibi et al. [27]. These in- 
vestigators studied IV transplantation of green fluorescent protein (GFP)-transduced 'MSCs' in immunocompetent neonatal mice. Early on, a wide tissue distribution of donor MSCs was seen with most of the cells lodged in the lung. This is the typical distribution of IV-infused MSCs as documented by numerous investigators. However, these investigators subsequently isolated BM cells from these mice using selection for GFP and demonstrated donor 'MSCs' in the bone and cartilage of recipient mice. While the authors interpreted this as proof of 'stemness' of the MSCs utilized, the only characterization of the cells was by GFP positivity and morphology of adherent cells in culture. The analysis provided of both the original 'MSC' population and the subsequently isolated repopulating cells, revealed a high proportion of CD45+ cells, making the conjecture that the engrafted cells that could be transplanted to secondary recipients were MSCs highly implausible.

Perhaps the best postnatal study examining the engraftment and differentiation potential of MSCs after IV infusion in a sub-lethal irradiation injury model is that of Anjos-Afonso et al. [28]. In contrast to the previous studies, the MSC population utilized were free of hematopoietic contamination and were unequivocally labeled by GFP transduction. As expected, the MSCs initially lodged in lung, and this is one of the few studies documenting pulmonary injury from MSC infusion. Subsequently, MSC-derived cells could be identified at very low frequency in a variety of tissues with, in some cases, documentation of morphology and immunohistochemistry consistent with differentiation into non-mesoderm-derived tissues such as bronchiolar epithelial, hepatocyte, and renal tubular cells. Importantly, the identified donor cells were in general single, with minimal evidence of in vivo clonal expansion, and the mechanism of differentiation was not defined, so the "differentiation' could have been by low frequency fusion events. Systemic administration has also been applied in a number of ischemic tissue and/or injury models, primarily models of CNS injury [29, 30], cardiac infarction [31-33], or spinal injury [34]. In the majority of such studies, functional improvement was documented but the mechanism of effect was not determined. While claims of in vivo neural differentiation have been made, the supporting evidence has been minimal, and at best, a very low frequency of in vivo differentiation to neural or cardiac cells has been demonstrated. In the majority of cases, the documented effects, if real, are likely due to indirect or paracrine mechanisms.

\section{Site-Specific Administration}

\section{Skeletal Tissue Repair}

The direct, site-specific injection of MSCs into tissues has successfully been utilized in several animal models to repair various connective tissue defects, particularly those involving cartilage and bone. Harris et al. [35] used fluorescent-labeled MSC-collagen gel constructs in a rabbit tendon model and found that the transplanted cells significantly improved tendon repair but also formed ectopic bone. Natsu et al. [36] studied the effects of BM-MSC transplantation in a rat muscle injury model and demonstrated enhanced tissue repair and muscle function of the injured muscle but were unable to demonstrate any differentiation or fusion of MSCs to muscle fibers. Yamada et al. [37] injected in vitro-expanded MSCs in a platelet-rich plasma (PRP) scaffold in an effort to increase the rate of bone formation in significant mandibular defects in dogs. The MSC-PRP combination was compared against injection of particulate cancellous bone marrow (PCBM). Results showed that by 8 weeks post-transplantation, defects filled with MSC-PRP were completely filled with normal bone macrostructure and abundant vascularization. This was in direct contrast to the PCBM-filled defects which demonstrated dead space within the defects, indicative of resorption. Although early results suggested an advantage of the MSC-PRP scaffold over PCBM in repairing large defects in bone, there was no significant difference in newly formed bone between these two treatment groups over time.

In a study carried out by Izuta et al. [38], GFP+ BM-MSCs embedded in fibrin glue were transplanted into the meniscal defects of Sprague-Dawley rats. The injured menisci were harvested and cultured for several weeks. By 12 weeks of culture, cartilage-like tissue could be observed surrounding fusiform cells in the transplanted group, signifying the production of an extracellular matrix by the MSCs. This finding was not observed in the control groups. Chen et al. [39] designed a study to investigate the effects of the normal joint cavity environment on the differentiation of autologous BM-MSCs into chondrocytes. Well characterized MSCs were expanded in vitro, fluorescently labeled, and implanted into the joint cavities of adult sheep. By 8 weeks after transplantation, implanted cells expressed type II collagen and synthesized sulfated proteoglycan; they did not express osteocalcin. These findings indicated that the implanted MSCs had not differentiated into osteoblasts. However, the researchers found that by co-culturing the MSCs with synovial fluid or synovial cells in vitro, chondrocytic differentiation could be accomplished in vivo, suggesting that environmental factors within the native joint are required for such differentiation.

Wan et al. [40] investigated the use of PB-MSCs to enhance bone regeneration in a rabbit ulna critical-sized bone defect model. PB was chosen as a source of MSCs due to the decreased morbidity of harvest, as compared to BM-MSCs. Ulna critical-sized bone defects created in 6-month-old rabbits were treated with PB-MSCs and compared to BM-MSC-treated defects and controls. 12 weeks after transplantation, bone formation efficiency was similar between both treatment groups. This finding was surprising, given that the colony-forming efficiency of BM-MSCs was found to be around 20 times higher than that of PB-MSCs.

A study carried out by Pagnotto et al. [41] demonstrated that induction of hMSCs to chondrogenesis by transforming 
growth factor- $\beta 1$ (TGF- $\beta 1$ ) led to improved cartilage repair. Of note, adenoviral-mediated transfer of TGF- $\beta 1$ has been shown to induce chondrogenesis while avoiding terminal differentiation of hMSCs [42]. In this study, adeno-associated virus (AAV) was used to transduce hBM-MSCs with TGF- $\beta 1$. These transduced cells were subsequently implanted into the osteochondral defects of athymic rats. Results demonstrated improved cartilage repair tissue in transplanted AAV-TGF$\beta 1$-transduced hMSCs by 12 weeks post-transplantation. Importantly, these effects persisted long-term (46 weeks).

\section{Myocardial Repair}

Great interest in cell transplantation to repair damaged myocardial tissue has been generated over the past several years. Various cell sources, including skeletal myoblasts, fibroblasts, cardiomyoctes, and BM cells, have been shown to engraft into cardiac tissue and improve function [43-45]. However, the potential for induction of immune response, as well as their finite supply has limited their use. Therefore, attention has been directed to the use of MSCs to repair myocardial ischemic injury. Though engraftment of donor MSCs into infracted myocardium has been well-established, controversy still exists over their cardiomyocyte differentiation and functional integration.

Grinnemo et al. [46] studied the survival and engraftment of hMSCs in a xenogeneic model of cardiac ischemia. hMSCs were directly injected into the myocardium of rats (with and without immunosuppression) 1 week after creation of a myocardial infarction (MI) by left anterior descending artery (LAD) ligation. hMSCs were shown to engraft in all groups, however, rats without immunosuppression showed evidence of transplant rejection, with infiltration of inflammatory cells. Furthermore, no differentiation into cardiomyocytes was demonstrated. Likewise, in a subsequent study by this group [47], hMSC transplantation into immunocompetent and T celldeficient immunoincompetent rats did not lead to functional improvement or cardiomyocyte differentiation, as evidenced by a lack of expression of various cardiomyocte markers (e.g. desmin, actin, myosin) by the transplanted hMSCs. Additionally, no transplanted hMSCs were found in immunocompetent recipients. Wang et al. [48], on the other hand, provided evidence of the successful engraftment and cardiogenic differentiation of allogeneic MSCs in a rabbit model of MI. The lowlevel differentiation of transplanted MSCs was established by tropomyosin inhibitory component (cTn1 expression), a marker for cardiomyocytes.

The angiogenic potential of MSC transplantation into peri-infarct areas has also been investigated; MSC-induced angiogenesis has been postulated to significantly contribute to the improved cardiac function seen with engraftment of MSCs into ischemic myocardium. Tang et al. [49] transplanted rat MSCs into the center of infracted myocardium 1 week after the induction of an MI. Angiogenesis was found to be significantly increased in the MSC group, as compared with controls. Addi- tionally, blood flow in the scar area was significantly higher in the former group. Hou et al. [50] investigated the engraftment of hMSCs from patients with coronary artery disease in a rat model of myocardial ischemia. Similar to results found by Tang et al. [49], significant angiogenesis was found to be induced by transplanted hMSCs.

In addition to the engraftment and differentiation potential of MSCs into ischemic myocardium, the effects of such transplantation on cardiac function has been extensively studied. Piao et al. [51] evaluated the functional effects of cultured allogeneic MSCs in a rat model of MI. Ten days after MI, rats received MSC injection into the border and center of the infarct scar. Subsequent echocardiographic examination revealed significant improvement of regional and global left ventricular function in the transplanted group. The improvements in cardiac function were thought to be mediated by improved blood flow in infracted myocardium, as a result of increased microvascular formation.

\section{Repair of Neural Injuries}

In the past, the majority of stem cell-based therapies in the treatment of CNS or spinal cord injuries have used either neural or embryonic stem cells. Though both of these stem cell populations have been shown to differentiate into neural cells in vivo, their broad application has been limited by their immunogenicity in host tissues, difficulty in obtaining sufficient amounts for transplantation, and ethical concerns, in the latter population. On the other hand, these issues do not pertain to adult MSCs. As such, the use of MSCs in the treatment of neural injuries has piqued the interest of several researchers, and promising, yet sometimes conflicting observations have been made.

Satake et al. [52] investigated the migration and differentiation of transplanted MSCs to sites of thoracic spinal cord injury (SCI). Adult male Lewis rats were transplanted with GFP-MSCs 3, 5, or 7 days after iatrogenic thoracic SCI; spinal cord tissue was harvested 1 or 2 weeks after MSC transplantation. Results indicated that transplanted MSCs migrated to the area of thoracic SCI and demonstrated neuronal stem cell differentiation, as evidenced by the expression of nestin in donor MSCs. However, specific cell markers for neurons and glial cells were not expressed, suggesting that full neuronal differentiation had not occurred at the time of analysis. Additionally, due to a low transfection rate in the transplanted cells, long-term follow-up was not accomplished. Bae et al. [53] used a Niemann-Pick type C mouse model (NP-C) to investigate the ability of BM-MSCs to promote the development of neuronal networks with functional synaptic transmission, by transplanting BM-MSCs into the brains of NP-C mice. These investigators found that BM-MSC transplantation significantly increased the number of surviving neurons in NP-C mice. More importantly, the neurons developed into electrically active Purkinje neurons with functional synaptic formation, as evidenced by increased levels of expression of excitatory glu- 
tamate receptors on Purkinje neurons. The researchers concluded that their results provided evidence that functional neurogenesis from BM-MSC transplantation is possible, at least within a neurodegenerative environment. However, such conclusions may be misleading. The transplanted BM-MSCs did not themselves generate new Purkinje neurons; rather, they fused with existing neurons, which resulted in regeneration of synaptic formation in the NP-C cerebellum. The ability of BM-MSCs to transdifferentiate into neurons was not demonstrated.

It has long been suggested that MSCs can only differentiate into neuronal cells after in vitro induction in appropriate media [54, 55]. Recently, several authors have demonstrated otherwise. Unlike the Bae study, full differentiation of transplanted MSCs was demonstrated in an experiment carried out by Zhang et al. [56]. In this study, fluorescently-labelled MSCs were used to treat rats that had experienced intracerebral hemorrhage (ICH). A well-characterized population of MSCs was transplanted via the carotid artery $(\mathrm{CA})$, cervical vein $(\mathrm{CV})$, or lateral ventricle (LV) into rats on varying days postICH. Rats were sacrificed and analyzed 7 days post-transplantation. Results demonstrated that MSCs transplanted via CA or $\mathrm{LV}$, but not $\mathrm{CV}$, migrated into injury sites of the brain. In addition, the transplanted MSCs differentiated into the various neuronal cell types, including neurons, astrocytes, and oligodendrocytes. Notable was the finding that transplanted MSCs expressed the neuronal cell markers nestin, NeuN, GFAP, and CNP in vivo, but not in vitro, indicating that there was no contamination of neural stems cells or neural cells in the cultured MSCs prior to transplantation. Deng et al. [57] evaluated the neural differentiation potential of male murine BM-MSCs in female neonatal mouse brains and found that such MSCs spontaneously express several neuronal markers (e.g. nestin, $\beta$-III tubulin) in vitro, without the aid of inductive media. Chromosomal analysis confirmed that the neuronal differentiation observed was in fact true MSC differentiation, as opposed to fusion of donor and host cells, a phenomenon previously reported in the literature.

Cizkova et al. [58] investigated the homing and engraftment of systemically transplanted hMSCs in an immunocompetent rat SCI model. hMSCs were transplanted via right femoral vein into immunocompetent rats 7 days after SCI. While transplanted hMSCs were found to differentiate into oligodendrocytes, no neuronal differentiation was demonstrated. Researchers concluded that the hMSCs most likely participated in remyelination of white matter tracts, as opposed to the regeneration of damaged neuronal tissues. In a similar rat SCI model, Lee et al. [59] examined the effects of hMSC transplantation on functional outcome of SCI rats. They found that the functional recovery of SCI rats was significantly improved compared to controls. Additionally, a few of the transplanted hMSCs were positive for neural cell markers, indicating lowlevel differentiation may have also contributed to the functional recovery of these animals.

\section{Wound Healing}

Acceleration of impaired wound healing is a clinically important goal. While the exogenous application of various growth factors, epidermal/dermal substitutes, and synthetic membranes have been thoroughly investigated, therapeutic effects have been limited. As such, recent attention has been placed on the use of MSCs to aid wound repair. Nakagawa et al. [60] studied the effects of hMSC transplantation on wound healing in a nude rat model. Skin tissue was excised from nude rats, and the defect was covered with a porcine artificial skin substitute that had been impregnated with hMSCs +/- basic fibroblast growth factor (bFGF) which has been reported to stimulate MSCs [61]. Results demonstrated that accelerated hMSC proliferation and wound healing occurred in experimental animals that received both hMSC and bFGF; this effect was bFGF concentration-dependent, with the optimal dose being $10 \mu \mathrm{g}$. Grafted hMSCs exhibited epithelial differentiation, as evidenced by the expression of human pancytokeratin (a re-epithelialization marker) in recipient wound beds. The in vivo epithelial differentiation potential of embryonic hMSCs has also been experimentally demonstrated. Wu et al. [62] subcutaneously injected GFP+ embryonic hMSCs into nude mice that did not have cutaneous defects. They found that transplanted embryonic hMSCs engrafted and differentiated into functional epidermal cells with production of both keratin 19 and E-cadherin.

Perng et al. [63] used human dermis-derived MSCs (hDMSCs) to facilitate wound healing in nude mice. Skin defects on the backs of nude mice were covered with hDMSCs on a gelatin scaffold. Wound sizes were recorded on a weekly basis. By day 7, the wound size of the hDMSC-treated group was significantly smaller than the control groups, and after 2 weeks the treated group had completely healed wounds. Additionally, expression of human pancytokeratin was significantly increased, indicating differentiation of hDMSCs into epithelial cells. Fu et al. [64] autografted well-characterized BM-MSCs to facilitate healing of full-thickness burn wounds in pigs. Transplanted MSCs were labeled with 5-bromodeoxyuridine (5-BrdU) to track proliferation and co-transplanted with either epidermal growth factor (EGF) or bFGF. Wound healing was significantly higher in the MSC treatment groups (MSC, MSC + EGF, MSC + bFGF) compared to the control group (no MSCs), though only low-level engraftment was demonstrated. 5-BrdU and pancytokeratin or factor VIII dualstained cells were observed, indicating donor MSC proliferation and transdifferentiation into epidermal and endothelial cells, though in very low numbers. More recently, an experimental model to test the healing potential of hMSCs in both human and murine acute and chronic cutaneous wounds was designed by Falanga et al. [65]. In human patients, cultured hMSCs were topically applied to acute (from skin cancer removal sites) and chronic (lower extremity vascular insufficiency) wounds. By 6 weeks post-transplant, the wounds had almost completely resurfaced. Immunostaining revealed differ- 
entiation into both fibroblasts and elastic fibers, the latter of which is not normally found in healing wounds or scars. The mouse wound model in this study was used to track fluorescently-labeled MSCs and see if they persisted long-term. Results demonstrated accelerated wound healing in the MSCtreated group, but the majority of cultured donor cells did not persist.

Finally, the effects of topical or locally injected MSCs have been assessed in animal models of impaired wound healing. There have been 2 recent studies in the impaired $\mathrm{db} / \mathrm{db}$ diabetic mouse model demonstrating accelerated wound healing with either BM- [66] or fetal liver-derived [67] murine MSCs. Both studies demonstrated a dramatic increase in angiogenesis and healing in MSC-treated wounds relative to controls. In contrast to the studies above, these studies found no evidence of epithelial or endothelial differentiation of the MSCs, but rather, MSCs appeared to act by direct and indirect paracrine mechanisms to enhance vascular ingrowth, wound granulation tissue formation, re-epithelialization, and production of extracellular matrix components to accelerate wound healing. Similar findings were observed using rabbit BM-derived MSCs in the ischemic rabbit ear model of impaired wound healing [68].

\section{Conclusions}

In summary, the evidence supporting in vivo differentiation of MSCs is relatively sparse and controversial compared to the abundance of data documenting in vitro multipotentiality. Most of the studies claiming in vivo differentiation, particularly beyond mesodermal-derived tissue types, have been methodologically flawed or have documented only extremely low frequency events. The entire field is lacking in vivo data supporting functional incorporation or tissue-specific gene expression of engrafted MSCs, and models of robust engraftment and incorporation do not exist. Nevertheless, many intriguing observations have been made that may eventually lead to fulfillment of the therapeutic promise of this unique and interesting cell population. In this pursuit, the prenatal models appear to offer unique advantages for both the engraftment and differentiation of MSC populations. These advantages include the immature immune status of the fetus that is permissive for engraftment of allogeneic and xenogeneic cells, the proliferative milieu of the early gestational fetus, and finally the ability to transplant cells during the formation of various tissue compartments, which may facilitate engraftment and provide specific inductive signals for in vivo differentiation.

\section{References}

1 Johnstone B, Hering TM, Caplan AI, et al: In vitro chondrogenesis of bone marrow-derived mesenchymal progenitor cells. Exp Cell Research 1998; 238:265-72.

2 Le Blanc K, Tammik L, Sundberg B, et al: Mesechymal stem cells inhibit and stimulate mixed lymphocyte cultures and mitogenic responses independently of the major histocompatibility complex. Scand J Immunol 2003;57:11-20.

3 Muller I, Kustermann-Kuhn B, Holzwarth C, et al: In vitro analysis of multipotent mesenchymal stromal cells as potential cellular therapeutics in neurometabolic disesases in pediatric patients. Exp Hematol 2006;34:1413-19.

$\checkmark 4$ Rieger K, Marinets O, Fietz T, et al: Mesenchymal stem cells remain of host origin even a long time after allogeneic peripheral blood stem cell or bone marrow transplantation. Exp Hematol 2005;33:605-11.

5 Wang J, Liu K, Lu DP: Mesenchymal stem cells in stem cell transplant recipients are damaged and remain of host origin. Int J Hematol 2005;82:152-8.

6 Almeida-Porada G, Hoffman R, Manalo P, et al Detection of human cells in human/sheep chimeric lambs with in vitro human stroma-forming potential. Exp Hematol 1996;24:482-7.

7 Almeida-Porada G, Flake AW, Glimp HA, Zanjani ED: Cotransplantation of stroma results in enhancement of engraftment and early expression of donor hematopoietic stem cells in utero. Exp Hematol 1999;27:1569-75.

$>8$ Almeida-Porada G, Porada CD, Tran N, Zanjani ED: Cotransplantation of human stromal cell progenitors into preimmune fetal sheep results in early appearance of human donor cells in circulation and boosts cell levels in bone marrow at later time points after transplantation. Blood 2000;95:3620-7. $\checkmark 9$ Liechty KW, MacKenzie TC, Shaaban AF, et al: Human mesenchymal stem cells engraft and demonstrate site-specific differentiation after in utero transplantation in sheep. Nat Med 2000;6: 1282-6.

10 Almeida-Porada G, Porada CD, Chamberlain J, et al: Formation of human hepatocytes by human hematopoietic stem cells in sheep. Blood 2004;104: 2582-90.

11 Chamberlain J, Yamagami T, Colletti E, et al: Efficient generation of human hepatocytes by the intrahepatic delivery of clonal human mesenchymal stem cells in fetal sheep. Hepatology 2007;46: 1935-45.

12 Almeida-Porada G, Porada CD, El Shabrawy D, et al: Human marrow stromal cells (MSC) represent a latent pool of stem cells capable of generating longterm hematopoietic cells. Blood 2001;98:713-9.

13 Almeida-Porada G, El Shabrawy D, Porada CD, et al.: Clonally derived MSC populations are able to differentiate into blood, liver and skin cells. Blood 2001;98:3292-8.

14 Guillot PV, Gotherstrom C, Chan J, et al: Human first-trimester fetal MSC express pluripotency markers and grow faster and have longer telomeres than adult MSC. Stem Cells 2007;35:646-54.

15 Airey JA, Almeida-Porada G, Colletti EJ, et al: Human mesenchymal stem cells form purkinje fibers in fetal sheep heart. Circulation 2004;109: 1401-7.

16 Colletti EJ, Almeida-Porada G, Chamberlain J, et al: The time course of engraftment of human mesenchymal stem cells in fetal heart demonstrates that Purkinje fiber aggregates derive from a single cell and not multi-cell homing. Exp Hematol 2006; 34:926-33
17 Chan J, Waddington S, O'Donoghue K, et al: Widespread distribution and muscle differentiation of human fetal mesenchymal stem cells after intrauterine transplantation in dystrophic $\mathrm{mdx}$ mouse. Stem Cells 2007;25:875-84.

18 Guillot PV, Abass O, Bassett JH, et al: Intrauterine transplantation of human fetal mesenchymal stem cells from first trimester blood repairs bone and reduces fractures in osteogenesis imperfecta mice. Blood 2008;111:1717-25.

19 Chou SH, Kuo TK, Liu M, Lee OK: In utero transplantation of human bone marrow-derived multipotent mesenchymal stem cells in mice. J Orthop Res 2006;24:301-12.

20 Munoz-Elias G, Marcus AJ, Coyne TM, et al: Adult bone marrow stromal cells in the embryonic brain engraftment, migration, differentiation, and longterm survival. J Neurosci 2004;24:4585-95.

21 Horwitz EM, Prockop DJ, Fitzpatrick LA, et al: Transplantability and therapeutic effects of bone marrow-derived mesenchymal cells in children with osteogenesis imperfecta. Nat Med 1999;5:309-13.

22 Horwitz EM, Prockop DJ, Gordon PL, et al: Clinical responses to bone marrow transplantation in children with severe osteogenesis imperfecta. Blood 2001;97:1227-31.

23 Horwitz EM, Gordon PL, Koo WK, et al: Isolated allogeneic bone marrow-derived mesenchymal cells engraft and stimulate growth in children with osteogenesis imperfecta: Implications for cell therapy of bone. Proc Natl Acad Sci U S A 2002;99:8932-7.

24 Bensidhoum M, Chapel A, Francois S, et al: Homing of in vitro expanded Stro-1- or Stro-1+ human mesenchymal stem cells into the NOD/SCID mouse and their role in supporting human CD34 cell engraftment. Blood 2004;103:3313-9. 
25 Mahmud N, Pang W, Cobbs C, et al: Studies of the route of administration and role of conditioning with radiation on unrelated allogeneic mismatched mesenchymal stem cell engraftment in a nonhuman primate model. Exp Hematol 2004;32:494-501.

26 Deng W, Han Q, Liao L, et al.: Allogeneic bone marrow-derived flk-1+ Sca-1- mesenchymal stem cells leads to stable mixed chimerism and donorspecific tolerance. Exp Hematol 2004:32:861-7.

27 Niyibizi C, Wang S, Mi Z, et al.: The fate of mesenchymal stem cells transplanted into immunocompetent neonatal mice: Implications for skeletal gene therapy via stem cells. Mol Ther 2004;9:955-63.

28 Anjos-Afonso F, Siapati EK, Bonnet D: In vivo contribution of murine mesenchymal stem cells into multiple cell-types under minimal damage conditions. J Cell Sci 2004;117:5655-64.

29 Mahmood A, Lu D, Chopp M: Intravenous administration of marrow stromal cells (MSCs) increases the expression of growth factors in rat brain after traumatic brain injury. J Neurotrauma 2004;21:33-9.

\$3 Mahmood A, Lu D, Qu C, Goussev A, Chopp M: Treatment of traumatic brain injury with a combination therapy of marrow stromal cells and atorvastatin in rats. Neurosurgery 2007;60:546-553; discussion 553-44.

31 Boomsma RA, Swaminathan PD, Geenen DL: Intravenously injected mesenchymal stem cells home to viable myocardium after coronary occlusion and preserve systolic function without altering infarct size. Int J Cardiol 2007;122:17-28.

32 Braga LM, Rosa K, Rodrigues B, et al: Systemic delivery of adult stem cells improves cardiac function in spontaneously hypertensive rats. Clin Exp Pharmacol Physiol. 2008;35:113-9.

-33 Noiseux N, Gnecchi M, Lopez-Ilasaca M, et al: Mesenchymal stem cells overexpressing Akt dramatically repair infarcted myocardium and improve cardiac function despite infrequent cellular fusion or differentiation. Mol Ther 2006;14:840-50.

34 Urdzikova L, Jendelova P, Glogarova K, Burian M, Hajek M, Sykova E: Transplantation of bone marrow stem cells as well as mobilization by granulocyte-colony stimulating factor promotes recovery after spinal cord injury in rats. J Neurotrauma 2006;23:1379-91.

35 Harris MT, Butler DL, Boivin GP, et al: Mesenchymal stem cells used for rabbit tendon repair can form ectopic bone and express alkaline phosphatase activity in constructs. J Ortho Res 2004;22: 998-1003.

36 Natsu K, Ochi M, Mochizuki Y, et al: Allogeneic bone marrow-derived mesenchymal stromal cells promote the regeneration of injured skeletal muscle without differentiation into myofibers. Tissue Eng 2004;10:1093-112.

\$3 Yamada Y, Ueda M, Naiki T, et al: Autogenous injectable bone for regeneration with mesenchymal stem cells and platelet-rich plasma: tissueengineered bone regeneration. Tissue Eng 2004;10: 955-64.

38 Izuta Y, Ochi M, Adachi N, et al: Meniscal repair using bone marrow-derived mesenchymal stem cells: experimental study using green fluorescent protein transgenic rats. The Knee 2005;12:217-223.
Chen J, Wang C, Lu S, et al: In vivo chondrogenesis of adult bone-marrow-derived autologous mesenchymal stem cells. Cell Tissue Res 2005;319:429-38.

40 Wan C, He Q, Li G: Allogenic peripheral blood derived mesenchymal stem cells (MSCs) enhance bone regeneration in rabbit ulna critical-sized bone defect model. J Orthop Res 2006;24:610-8.

41 Pagnotto MR, Wang Z, Karpie JC, et al: Adenoassociated viral gene transfer of transforming growth factor- $\beta 1$ to human mesenchymal stem cells improves cartilage repair. Gene Therapy 2007;14: 804-13.

42 Kawamura K, Chu CR, Sobajima S, et al: Adenoviral-mediated transfer of TGF- $\beta 1$ but not IGF-1 induces chondrogenic differentiation of human mesenchymal stem cells in pellet cultures. Exp Hematol 2005;33:865-72.

43 Sakai T, Li RK, Weisel RD, et al: Fetal cell transplantation: a comparison of three cell types. J Thorac Cardiovasc Surg 1999;118:715-25.

44 Li RK, Weisel RD, Mickel DA, et al: Autologous porcine heart cell transplantation improved heart function after a myocardial infarction. J Thorac Cardiovasc Surg 2000;119:62-8.

45 Toma C, Pittenger MF, Cahill KS, et al: Human mesenchymal stem cells differentiate to a cardiomyocyte phenotype in the adult murine heart. Circulation 2002;105:93-8.

46 Grinnemo KH, Mansson A, Dellgren G, et al: Xenoreactivity and engraftment of human mesenchymal stem cells transplanted into infracted rat myocardium. J Thorac Cardiovasc Surg 2004;127: 1293-300.

47 Grinnemo KH, Mansson-Broberg A, Leblanc K, et al: Human mesenchymal stem cells do not differentiate into cardiomyocytes in a cardiac ischemic xenomodel. Ann Med 2006;38:144-53.

48 Wang J, Li C, Fan Y, et al: Allograftic bone marrowderived mesenchymal stem cells transplanted into heart infracted model of rabbit to renovate infracted heart. J Zhejiang Univ SCI 2004;5:1279-85.

49 Tang YL, Zhao Q, Zhang YC, et al: Autologous meesnchymal stem cell transplantation induce VEGF and neovascularization in ischemic myocardium. Regulatory Peptides 2004;117:3-10.

50 Hou M, Yang K, Zhang H, et al: Transplantation of mesenchymal stem cells from human bone marrow improves damaged heart function in rats. Int $\mathbf{J}$ Cardiol 2007;115:220-8.

51 Piao H, Youn TJ, Kwon JS, et al: Effects of bone marrow derived mesenchymal stem cells transplantation in acutely infracting myocardium. Eur J Heart Failure 2005;7:730-8.

52 Satake K, Lou J, Lenke LG: Migration of mesenchymal stem cells through cerebrospinal fluid into injured spinal cord tissue. Spine 2004;29:1971-9.

53 Bae JS, Han HS, Youn DH, et al: Bone marrowderived mesenchymal stem cells promote neuronal networks with functional synaptic transmission after transplantation into mice with neurodegeneration. Stem Cells 2007;25:1307-16.

54 Lu P, Blesch A, Tuszynski MH: Induction of bone marrow stromal cells to neurons: differentiation, trans-differentiation, or artifact? J Neurosci Res 2004;77:174-91.
55 Neuhuber B, Gallo G, Howard L, et al: Reevaluation of in vitro differentiation protocols for bone marrow stromal cells: disruption of actin cytoskeleton induces rapid morphological changes and mimics neuronal phenotype. J Neurosci Res 2004; 77:192-204.

56 Zhang H, Huang Z, Xu Y, et al: Differentiation and neurological benefit of the mesenchymal stem cells transplanted into the rat brain following intracerebral hemorrhage. Neurol Res 2006;28:104-12.

57 Deng J, Petersen BE, Steindler DA, et al: Mesenchymal stem cells spontaneously express neural proteins in culture and are neurogenic after transplantation. Stem Cells 2006;24:1054-64.

58 Cizkova D, Rosocha J, Vanicky I, et al: Transplants of human mesenchymal stem cells improve functional recovery after spinal cord injury in the rat. Cell Mol Neurobiol 2006;26:1167-80.

59 Lee KH, Suh-Kim H, Choi JS, et al: Human mesenchymal stem cell transplantation promotes functional recovery following acute spinal cord injury in rats. Acta Neurobiol Exp 2007;67:13-22.

60 Nakagawa H, Akita S, Fukui M, et al: Human mesenchymal stem cells successfully improve skin-substitute wound healing. Br J Dermatol 2005;153: 29-36.

61 Akino K, Mineta T, Fukui, et al: Bone morphogenetic protein-2 regulates proliferation of human mesenchymal stem cells. Wound Repair Regen 2003;11:354-60.

$62 \mathrm{Wu}$ M, Yang S, Liu H, et al: Differentiation potential of human embryonic mesenchymal stem cells for skin-related tissue. Br J Derm 2006;155:282-91.

63 Perng CK, Ku HH, Chiou SH, et al: Evaluation of wound healing effect on skin-defect nude mice by using human dermis-derived mesenchymal stem cells. Transplant Proc 2006;38:3086-7.

64 Fu X, Fang L, Li X, et al: Enhanced wound-healing quality with bone marrow mesenchymal stem cell autografting after skin injury. Wound Repair Regen 2006;14:325-35.

65 Falanga V, Iwamoto S, Chartier M, et al: Autologous bone marrow-derived cultured mesenchymal stem cells delivered in a fibrin spray accelerate healing in murine and human cutaneous wounds. Tissue Eng 2007;13:1299-312.

66 Javazon EH, Keswani SG, Badillo AT, et al: Enhanced epithelial gap closure and increased angiogenesis in wounds of diabetic mice treated with adult murine mesenchymal stem cells. Wound Repair Regen 2007;15:350-9.

67 Badillo AT, Redden RA, Zhang L, Doolin EJ, Liechty KW: Treatment of diabetic wounds with fetal murine mesenchymal stromal cells enhances wound closure. Cell Tissue Res 2007:329:301-11.

68 Volk SW, Radu A, Zhang L, Liechty KW: Stromal progenitor cell therapy corrects the wound-healing defect in the ischemic rabbit ear model of chronic wound repair. Wound Repair Regen 2007;15:736-47. 